Original Article (short paper)

\title{
Effects of aerobic, anaerobic, and concurrent training on bone mineral density of rats
}

\author{
Robson Chacon Castoldi \\ Universidade Estadual de Campinas, Campinas, SP, Brasil \\ Mario Jefferson Quirino Louzada \\ Bruna Rezende Silva Martins de Oliveira \\ Universidade Estadual Paulista “Júlio de Mesquita Filho”, Araçatuba, SP, Brasil \\ Guilherme Akio Tamura Ozaki \\ Universidade Estadual de Campinas, Campinas, SP, Brasil \\ Tatiana Emy Koike \\ Thiago Alves Garcia \\ Regina Celi Trindade Camargo \\ Universidade Estadual Paulista "Júlio de Mesquita Filho", Presidente Prudente, SP, Brasil \\ William Dias Belangero \\ Universidade Estadual de Campinas, Campinas, SP, Brasil \\ José Carlos Silva Camargo Filho \\ Universidade Estadual Paulista “Júlio de Mesquita Filho", Presidente Prudente, SP, Brasil
}

\begin{abstract}
A sample of 39 rats were divided into the following groups: baseline (BL); aerobic training (ET4 and ET8); anaerobic training (ST4 and ST8); and concurrent training (CT4 and CT8). The aerobic training was performed by swimming with a load corresponding to $70 \%$ of the anaerobic threshold; the anaerobic training was performed by jumping in water with a load corresponding to $50 \%$ of body weight; and the concurrent training combined the two protocols. The analysis of BMD was performed on the tibia of the animals and showed an increase in the density of the ST4, CT4, ET8, ST8, and CT8 groups when compared with both the BL and ET4. Thus, it is concluded that anaerobic exercise was shown to be effective in increasing the BMD of animals after four weeks of training; however, aerobic training was only able to raise BMD following eight weeks of training.
\end{abstract}

Keywords: bone density, aerobic exercise, physical exercise.

\section{Introduction}

Physical training is important for maintaining bone mass as it acts by means of mechanical and metabolic stimuli, modeling the synthesis of the bone matrix ${ }^{1}$. However, training presents variations in method of application with differences in duration and intensity that promote different stimuli, both mechanical and metabolic ${ }^{2}$.

Among the various classifications of physical training, aerobic and anaerobic are highlighted and defined according to their metabolic dominance. Aerobic training presents a low-intensity and long-duration, differing from anaerobic training, which is characterized by a high-intensity and short-duration ${ }^{3}$.

Aerobic training is widely used for the treatment of osteoporosis as it presents a low-risk of fractures due to the low-intensity combined with metabolic stimulus necessary for bone synthesis, while anaerobic training presents significant mechanical stimulation due to its high-intensity ${ }^{4}$.
Increased bone synthesis increases bone mineral density by increasing synthesis of hydroxyapatite crystals in the bone matrix $^{1,2}$. The method used to quantify bone mineral density (BMD) is dual energy X-ray absorptiometry (DXA), which quantifies the mineral content of the bone $\mathrm{e}^{2,5}$.

Concurrent training was conducted with the two exercise modalities - aerobic and anaerobic - in the same training session. The effects of this type of training present disagreement in the literature with studies that show beneficial effects, harmful effects, and no effects ${ }^{3,6}$.

The use of concurrent training could promote increased bone mineral density, combining the effects of aerobic and anaerobic training and serve as a resource for new interventions in populations at risk of diseases, such as osteoporosis, and could assist in growth phases during which bone tissue develops in children and young people $\mathrm{e}^{3,6,7}$.

Therefore, the present study aimed to evaluate the effects of aerobic, anaerobic, and concurrent training on the bone mineral density of rats. 


\section{Method}

\section{Sample}

In total, 39 rats were used (Wistar). The animals were 100 days' old and obtained from the Central Animal Laboratory of Unesp, Botucatu -SP. They were kept in the small animal vivarium at the Histology and Histochemistry Laboratory, Physical Therapy Department, Faculty of Science and Technology - FCT/UNESP, Presidente Prudente Campus - SP. The animals were placed in collective cages with up to five animals per cage (polyethylene) with an ambient temperature $\left(22 \pm 2^{\circ} \mathrm{C}\right), 12 \mathrm{hr}$ light/dark cycle, humidity of $50 \pm 10 \%$, and were fed standard chow and water ad libitum.

All procedures were previously approved by the Ethics Committee on Animal Use of FCT/UNESP under protocol number 002/2011 in accordance with regulations established by the Brazilian College of Animal Experimentation (COBEA).

\section{Experimental groups}

The animals were divided into groups according to the training protocol and training period of four or eight weeks as follows: baseline, BL $(\mathrm{n}=5)$; aerobic training ET4 $(\mathrm{n}=6)$ and ET8 $(\mathrm{n}=7)$; anaerobic training ST4 $(\mathrm{n}=6)$ and ST8 $(\mathrm{n}=5)$; and concurrent physical training CT4 $(n=5)$ and CT8 $(n=5)$.

\section{Experimental design}

The experiment began with a determination of the training load through the Critical Workload test (CL). After defining the loads, the animals were submitted to aerobic, anaerobic, or concurrent training, and were euthanized after the training period. The animals of the BL group were euthanized immediately prior to initiation of the experimental period.

\section{Critical Workload Test}

Determination of the aerobic capacity, by means of the critical load, was obtained by inducing exercise in four different stimuli, which were randomized with loads of $7 \%, 9 \%, 11 \%$, and $13 \%$ of the body weight so that all animals performed the four efforts and reached exhaustion after between two and ten minutes, as proposed by $\mathrm{Hill}^{8}$. However, due to the failure of the animals at the $13 \%$ body weight stimulation, this intensity was excluded from the determination of the anaerobic threshold.

The time taken to perform the exercise at each load was timed using a stopwatch (TIMEX ${ }^{\circledR}$, model 85103). Every effort was performed at intervals of 24 hours, following the method of Chimin et al. ${ }^{9}$. Next, a linear regression was performed between the load and inverse of the time for each effort $(1 /$ tlim), from which an equation was generated to verify the value corresponding to the aerobic capacity, represented by the variable $b$ in the equation (Figure 1).

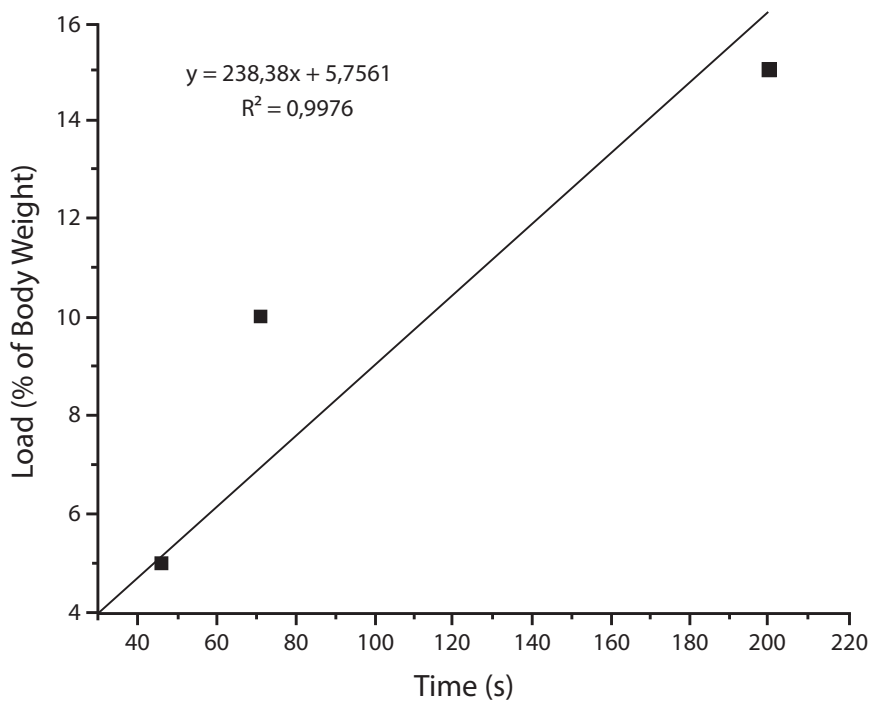

Figure 1: CL scatter plot with real values of the test performed by one animal. In this case, the Lan corresponded to $\approx 5.76 \%$ of the BW.

\section{Aerobic training protocol}

For the aerobic training, the animals were submitted to 30 minutes of swimming, three times a week, in appropriate tanks subdivided by PVC cylinders to individualize the lanes so that each animal trained individually. The intensity was equivalent to $70 \%$ of the anaerobic threshold (AT), set by the Critical Workload test ${ }^{9}$.

\section{Anaerobic training protocol}

The anaerobic training was composed of four sets of 10 jumps, three times per week, in a cylindrical PVC container specially modified for jumps in water (50 cm long) with an appropriate depth for the size of the animals ( $38 \mathrm{~cm}$ deep). Between each series of jumps, a 1-minute interval was established and verified by a stopwatch. The overload used corresponded to $50 \%$ of the body weight of each animal, accommodated on the anterior chest by means of a vest ${ }^{10}$.

\section{Concurrent training protocol}

The concurrent training combined the two physical training models mentioned above. The exercise sessions were performed in sequence (aerobic and anaerobic training) without an interval between them, three times a week, comprising 30 minutes of swimming (load set to $70 \%$ Lan) and four series of 10 jumps with an overload of $50 \%$ of the body weight of each animal ${ }^{3}$.

\section{Collection and preparation of material}

Following the experimental period, the animals were euthanized through an overdose of an association of ketamine hydrochloride and xylazine, intraperitoneally, following ethical principles in animal research. 
The right tibias of the animals were removed by surgical procedure and then immersed in saline solution and stored in a conventional freezer $\left(-20^{\circ} \mathrm{C}\right)$ for subsequent DXA analysis.

\section{$D X A$}

To perform the DXA analysis, the tibias were first thawed at room temperature and then submitted to densitometry analysis in the DXA, DPX-Alpha model, LUNAR $\AA$, belonging to the Aracatuba Faculty of Veterinary Medicine - FMVA/UNESP, using special software for small animals ${ }^{2,10}$.

The DXA analysis was based upon the measurement of the following parameters: total area of bone; Bone Mineral Content (BMC) and the relationship between BMC and area; and denominated Bone Mineral Density (BMD).

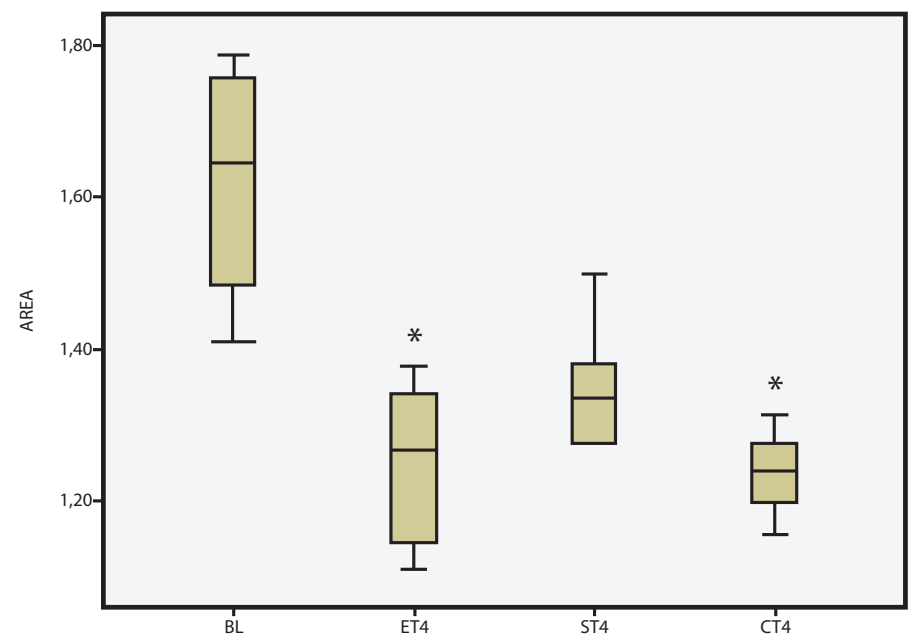

\section{Statistical Analysis}

Data were analyzed using the Shapiro-Wilk test, which confirmed the Gaussian distribution of the data, after which oneway ANOVA followed by Tukey's post-test were used to verify the differences between the groups. The tests were performed using SPSS 17 software with an adopted significance level of $5 \%(\mathrm{p}<0.05)$.

\section{Results}

The analysis of the area $\left(\mathrm{cm}^{2}\right)$ of the bones demonstrated lower values in the ET4 and CT4 groups in relation to the $\mathrm{BL}$; there were no significant alterations between the other groups (Figure 2).

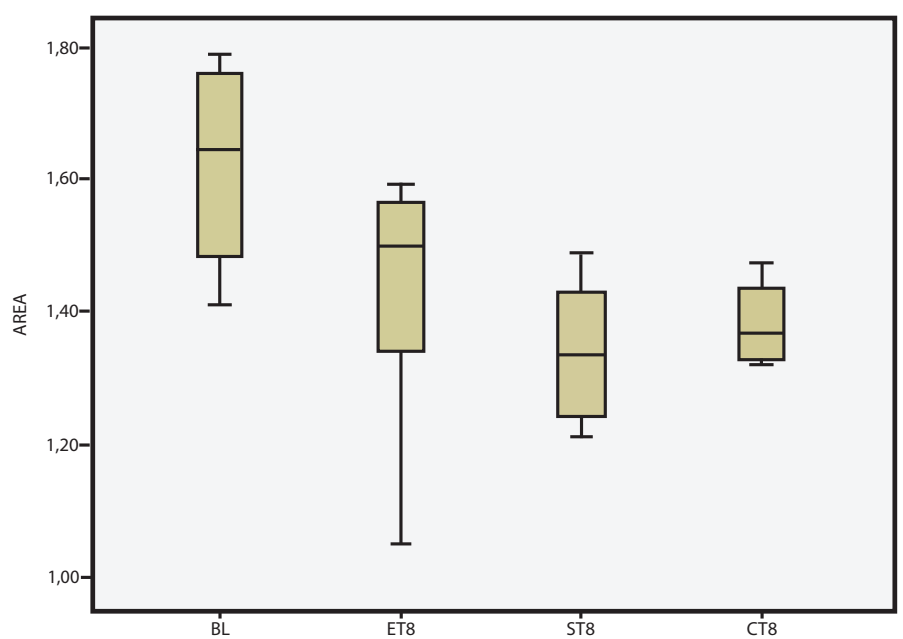

Figure 2: Box-plot with values of area in $\mathrm{cm}^{2}$. * Significant difference in relation to the BL group.

Evaluation of the BMC (g) showed a significant increase in the ST4, ET8, ST8, and CT8 groups when compared to the ET4 group (Figure 3).

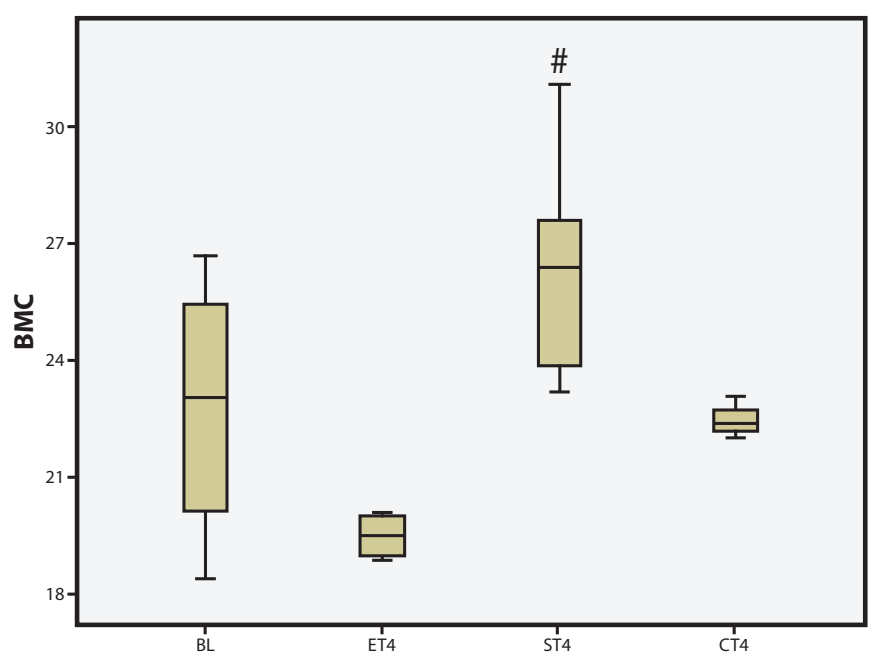

The analysis of BMD $\left(\mathrm{g} / \mathrm{cm}^{2}\right)$ presented an increase in the density of the ST4, CT4, ET8, ST8, and CT8 groups compared with both the BL and ET4 groups (Figure 4).

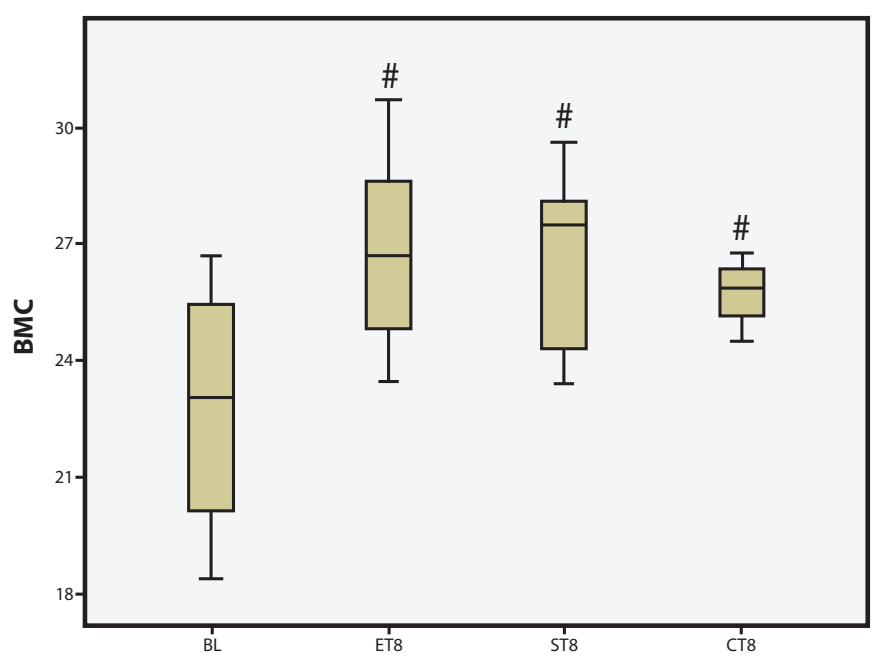

Figure 3: Box-plot with the values of BMC (g). \# Significant difference in relation to the ET4 group. 

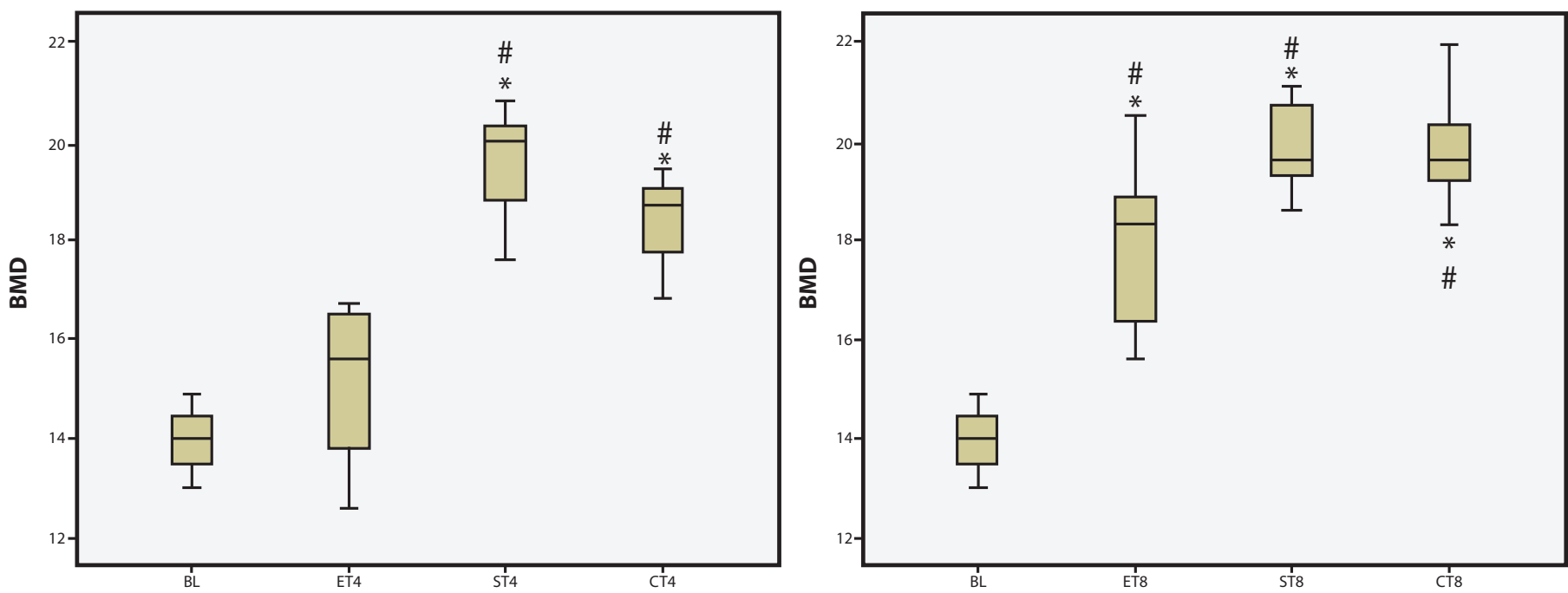

Figure 4: Box-plot with the values of BMD $\left(\mathrm{g} / \mathrm{cm}^{2}\right) .{ }^{*}$ Significant difference in relation to the BL group; \# Significant difference in relation to the ET4 group.

\section{Discussion}

The results demonstrate that four weeks of aerobic training failed to increase BMD compared to the BL group, differing from the anaerobic and concurrent training, which significantly increased the BMD compared to the BL group. With respect to the eightweek period, all the training models significantly increased BMD in relation to the BL group, with no differences between the training methods in this period. However, no differences were observed between the anaerobic and concurrent training in either of the two training periods (ST4, ST8, CT4, CT8).

Previous studies ${ }^{2,11}$ have shown that the absence of mechanical stimuli, by means of immobilization or suspension of the animal, results in a reduction in BMD and fracture resistance. In contrast, physical exercise can act as an important non-drug intervention, increasing BMD and bone tissue resistance. Exercises with no impact, such as swimming and cycling, do not demonstrate an effect on improving $\mathrm{BMD}^{12,13}$; however, studies that used swimming to reverse a BMD reduction framework caused by the absence of mechanical stimuli showed effectiveness in increasing BMD after swimming exercise $e^{2,12,14}$.

In the present study, although there was no difference between the ET4 and BL groups, the animals that performed the aerobic swimming exercise demonstrated increased BMD after eight weeks of training (ET8), compared to the BL group without intervention. Thus, swimming exercise, while not subjecting the bone tissue to impact, demonstrated an osteogenic effect, although of lesser magnitude since it was only able to increase BMD after eight weeks of training ${ }^{12,13}$.

Calcium deposition in bone is partly regulated by the mechanical stress to which the bone is subjected ${ }^{2}$. Although the mechanism involved in bone anabolism is not yet completely clear, it is known that a mechanical stimulus acts both in the inhibition of bone resorption through the action of NO (nitric acid), which promotes osteoclast inhibition, and in bone synthesis by increasing the intracellular calcium in osteoblasts and serum levels of IGF-1, factors that increase BMD and bone strength ${ }^{15,16}$.
In addition, exercise promotes metabolic and hormonal alterations that also stimulate the synthesis of calcium in bone tissue ${ }^{15-17}$.

Thus, the aquatic exercise-induced increase in mineral density (ET8 group) could be explained by the mechanical stress caused by the tendon traction on the bones during muscle contraction, together with the metabolic and hormonal alterations caused by exercise.

Therefore, it is suggested that this type of exercise can be used in conditions of severe loss of bone mass as a method of treatment, especially in cases with a high-risk of fractures, as the reduced impact on the bones from this exercise modality makes practitioners less susceptible to bone fractures ${ }^{12,17}$.

With respect to the anaerobic training, it presented higher osteogenic potential than aerobic training (swimming) since after just four weeks, it was able to significantly increase BMD with respect to the aerobic swimming training groups (ET4) and the group without training (BL), and the same occurred with concurrent training. In this way, the osteogenic effect of the water jump (anaerobic training) was demonstrated to be effective, even when combined with exercise without impact (aerobic swimming training $)^{18}$. In addition, training with no impact was demonstrated not to be detrimental to BMD, since although it did not increase BMD, it also did not induce significant losses in the deposition of calcium in the bone, and did not detract from the osteogenic effect when combined with anaerobic jump training (concurrent group) ${ }^{1,2,6}$.

To increase BMD, exercises that provide impact on the musculoskeletal system are recommended, as calcium is deposited in bone tissue in response to tissue deformation. Hence, the greater the magnitude of the applied forces, the greater the stimulation of osteoblasts to synthesize calcium in the bone matrix $^{2,19}$. Therefore, exercises with impact present a greater osteogenic effect, as in addition to alterations in the metabolism generated by exercise, they also act through mechanoreceptors, further stimulating bone synthesis ${ }^{1,20}$.

The present study was limited to evaluating the effects of training on bone tissue, although other factors also influence 
BMD, such as the condition of nutrition, these factors were not measured. Moreover, studies evaluating BMD in pathological conditions, such as osteoporosis, would be of great importance.

\section{Conclusion}

It is concluded that anaerobic exercise was shown to be effective in increasing the BMD of animals after four weeks of training; however, aerobic training (swimming) was only able to increase BMD after eight weeks of training.

\section{References}

1. Knapik DM, Perera P, Nam J, Blazek AD, Rath B, Leblebicioglu $\mathrm{B}$, et al. Mechanosignaling in bone health, trauma and inflammation. Antioxid Redox Signal. 2014;20(6):970-85.

2. Ozaki GAT, Koike TE, Castoldi RC, Garçon AAB, Kodama FY, Watanabe AY, et al. Efeitos da remobilização por meio de exercício físico sobre a densidade óssea de ratos adultos e idosos. Motricidade. 2014 Sep 1 [cited 2014 Dec 4];10(3):71-8.

3. Castoldi RC, Camargo RCT, Magalhães AJB, Ozaki GAT, Kodama FY, Oikawa SM, et al. Concurrent training effect on muscle fibers in Wistar rats. Mot Rev Educ Física. 2013;19(4):717-23.

4. Santos ML dos, Borges GF. Exercício físico no tratamento e prevenção de idosos com osteoporose: uma revisão sistemática. Fisioter em Mov. 2010;23(2):289-99.

5. McVeigh J, Kingsley S, Gray D, Loram LC. Swimming enhances bone mass acquisition in growing female rats. J Sport Sci Med. 2010;9(4):612-9.

6. Frajacomo FTT, Falcai MJ, Fernandes CR, Shimano AC, Garcia SB. Biomechanical adaptations of mice cortical bone submitted to three different exercise modalities. Acta Ortop Bras. 2013;21(6):328-32.

7. Ferry B, Duclos M, Burt L, Therre P, Le Gall F, Jaffré C, et al. Bone geometry and strength adaptations to physical constraints inherent in different sports: comparison between elite female soccer players and swimmers. J Bone Miner Metab. 2011;29(3):342-51.

8. Hill D. The critical power concept. A review. Sport Med. 1993;16(4):237-54.

9. Chimin P, Araújo GG, Macnchado-Gobatto FB, Gobatto CA. Critical load during continuous and discontinuous training in swimming Wistar rats. Motricidade. 2009;5(4):45-58.

10. Apolinário J de C, Coelho WMD, Louzada MJQ. Análise da influência do ultrassom de baixa intensidade na região de reparo ósseo em ratos sob ausência de carga Analysis of the influence of low-intensity ultrasound the region of bone repair in rats under no load. Fisioter e Pesqui. 2011;18(3):275-9.

11. Benda NMM, Seeger JPH, Stevens GGCF, Hijmans-Kersten BTP, van Dijk APJ, Bellersen L, et al. Effects of High-Intensity Interval Training versus Continuous Training on Physical Fitness, Cardiovascular Function and Quality of Life in Heart Failure Patients. PLoS One. 2015;10(10):e0141256.
12. Gómez-Bruton A, Gónzalez-Agüero A, Gómez-Cabello A, Casajús JA, Vicente-Rodríguez G. Is bone tissue really affected by swimming? A systematic review. PLoS One. 2013;8(8):e70119.

13. Paliologo T, Shimano RC, Shimano AC, Macedo AP, Falcai MJ, Issa JPM. Effects of swimming associated with risedronate in osteopenic bones: An experimental study with ovariectomized rats. Micron. 2015 Nov;78:40-4.

14. Ju Y-I, Sone T, Ohnaru K, Tanaka K, Fukunaga M. Effect of swimming exercise on three-dimensional trabecular bone microarchitecture in ovariectomized rats. J Appl Physiol. 2015 Nov 1;119(9):990-7.

15. Falcai MJ, Zamarioli A, Okubo R, de Paula FJA, Volpon JB. The osteogenic effects of swimming, jumping, and vibration on the protection of bone quality from disuse bone loss. Scand J Med Sci Sports. 2015 Jun;25(3):390-7.

16. Turner CH. Bone strength: current concepts. Ann N Y Acad Sci. 2006 Apr;1068(1):429-46.

17. Volpon JB, Silva AV, Falcai MJ, Louzada MJQ, Zamarioli A, Kotake BGDS, et al. The osteogenic effects of swimming on bone mass, strength, and microarchitecture in rats with unloadinginduced bone loss. Microsc Res Tech. 2015 Sep;78(9):784-91.

18. Mosavat M, Ooi FK, Mohamed M. Stress hormone and reproductive system in response to honey supplementation combined with different jumping exercise intensities in female rats. Biomed Res Int.2014;(1):123640.

19. Val FF de A e, Okubo R, Falcai MJ, Asano FS, Shimano AC. Effects of high-impact exercise training on bone mechanical proprieties-an experimental study in female wistar rats. Rev Bras Med do Esporte. 2013;19(4):252-5.

20. Shanb AA, Youssef EF. The impact of adding weight-bearing exercise versus nonweight bearing programs to the medical treatment of elderly patients with osteoporosis. J Family Community Med. 2014;21(3):176-81.

\section{Acknowledgments}

Sources of funding: Coordination for the Improvement of Higher Education Personnel (CAPES).

\section{Corresponding author}

Guilherme Akio Tamura Ozaki

Doutorando em Ciências da Cirurgia, Unicamp, SP. Professor e técnico de Karatê do município de Presidente Prudente, SP. Graduado e Mestre em Fisioterapia pela Faculdade de Ciências e Tecnologia, FCT/UNESP. LABORATÓRIO DE ANÁLISE DA PLASTICIDADE MUSCULAR (LAPMus), Faculdade de Ciências e Tecnologia, FCT/UNESP, Campus de Presidente Prudente, SP.

Rua Roberto Simonsen, 305, Presidente Prudente, SP

Email: guiozaki@hotmail.com

Manuscript received on July 22, 2016

Manuscript accepted on October 25, 2016

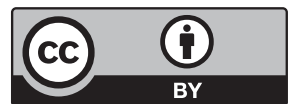

Motriz. The Journal of Physical Education. UNESP. Rio Claro, SP, Brazil - eISSN: 1980-6574 - under a license Creative Commons - Version 3.0 